\title{
PALEOGRAPHIE COMPAREE DES ECRITURES EPIGRAPHIQUES ET MANUSCRITES : UNE METHODOLOGIE EN EVOLUTION
}

\section{COMPARED PALAEOGRAPHY OF EPIGRAPHIC AND MANUSCRIPT WRITINGS: TESTING OF A METHODOLOGY}

EVA CARAMELLO

Université de Poitiers

Résumé : Les épigraphistes français depuis quelques décennies reconnaissent régulièrement l'importance de l'étude des caractères extérieurs d'une inscription ; cependant encore aujourd'hui ils ont du mal à leur donner la juste place au sein de l'analyse des textes épigraphiques médiévaux. Néanmoins l'approche paléographique d'un texte gravé est toujours plus souhaitée, surtout grâce à la diffusion d'une pluridisciplinarité entre les sciences auxiliaires de l'histoire, mais pas encore régulièrement appliquée. La conséquence directe de l'absence d'une vraie tradition paléographique appliquée aux écritures épigraphiques est que la paléographie n'est pas considérée scientifiquement. À partir des résultats solides offerts de la paléographie traditionnelle, cette recherche en cours voudrait ici proposer une méthode d'analyse des caractères graphiques présents dans les inscriptions, méthode qui se veut exemplaire et applicable pour l'étude de n'importe quel autre corpus.

Parce qu'il s'agit d'une approche innovante, le travail à faire n'est pas toujours si évident : à partir de la création des outils pour accomplir la recherche (vocabulaire technique pour décrire la lettre gravée, grille d'analyse, tableaux chronologiques) jusqu'au manque d'études systématiques, il s'agit d'une démarche à créer intégralement. En profitant de la paléographie in stricto sensu, soit pour sa méthode d'investigation, soit en comparant les écritures d'apparat des manuscrits avec les écritures épigraphiques, nous chercherons à faire de l'étude des écritures gravées un critère scientifique ponctuellement employé par les spécialistes. Nous avons choisi le corpus d'inscriptions et de manuscrits offert par la ville de Toulouse dans une chronologie comprise entre les $\mathrm{XI}^{\mathrm{e}}-\mathrm{XIV}^{\mathrm{e}}$ siècles, puisqu'il est un des rares exemples français de production, «en série », parfait donc pour la démonstration d'une méthodologie.

Mots-Clé : Paléographie comparée, épigraphie, écriture d'apparat, $\mathrm{XI}^{\mathrm{e}}$ siècle, $\mathrm{XIV}^{\mathrm{e}}$ siècle, France. 
Abstract: French Epigraphists in recent decades regularly recognize the importance of studying the external characteristics of an inscription; however they still have a hard time giving them the proper place in the analysis of medieval epigraphic texts. Nevertheless the approach of a paleographic engraved text is always more desired, especially through the dissemination of a multidisciplinary approach between the auxiliary sciences of history, but it is not yet consistently applied. Paleography is not yet considered scientifically, because of the absence of a real paleographic tradition in the study of epigraphic writings. Based on the results of the available solid traditional paleography, this current research would suggest here a method of analysis of the graphic characters present in the inscriptions, a method that want to be exemplary and applicable for the study of any other corpus of inscriptions.

Because it is an innovative approach, the works is not always so obvious: from the creation of tools to do research (technical vocabulary to describe the letter engraved, analytical, timelines) to the lack of systematic studies in this field, we have to create this approach entirely. By taking advantage of paleography in the strict sense, either for its method of investigation, or comparing the writings of titles in epigraphic manuscripts with the books, we will seek to make the study of scriptures carved a scientific criterion duly used by specialists. We chose the corpus of inscriptions and manuscripts offered by the city of Toulouse in a timeline between the 11th and 14th centuries, since it is a rare example of French "serial" production, so perfect for the demonstration of methodology.

Keywords: Comparative paleography, Epigraphy, Title writings, 11th century, 14th century, France.

La rareté des études sur la paléographie lapidaire est principalement due à deux motifs : d'abord parce que l'écriture épigraphique, antique, médiévale ou moderne est par nature "facile" à déchiffrer dans la mesure où elle doit s'adresser au plus grand nombre de lecteurs, y compris ceux dont la familiarité avec les phénomènes écrits est limitée ; en deuxième lieu parce qu'elle se caractérise par une forme de constance sur la longue durée autour des formes majuscules, amenant de fait les paléographes à s'occuper principalement des graphies cursives et/ou minuscules présentes surtout dans les chartes et les manuscrits.

Ce n'est qu'à partir de la seconde moitié du $\mathrm{XX}^{\mathrm{e}}$ siècle que l'on relève une nouvelle sensibilité pour l'écriture épigraphique qui conduit aux premières collaborations entre paléographes et épigraphistes ${ }^{1}$. Leurs intérêts se limitent pourtant

${ }^{1}$ R. MARICHAL, "Paléographie et épigraphie latine", dans Actes du IIe congrès international d'épigraphie grecque et latine, 1952. J. DURLIAT, "Écritures écrites et écritures épigraphiques. Le dossier des inscriptions byzantines d'Afriques", dans Studi medievali, 3 e série, 21, 
souvent à l'étude d'un groupe d'écritures manuscrites déjà bien connues et nouvellement confrontées à une analyse du point de vue épigraphique. De ces premières confrontations est née une bibliographie partielle et trop limitée, réduite presque toujours à l'étude des cas individuels, et ne proposant pas de schémas solides pour l'évolution de la graphie des textes lapidaires, laissant ainsi des vides très importants pour les études contemporaines des phénomènes graphiques.

Les recherches que je mène actuellement dans le cadre d'une thèse de doctorat naissent d'une volonté nouvelle de considérer l'écriture ancienne comme un phénomène global d'une société, indépendamment du support sur lequel elle est présente, en suivant l'enseignement, entre autres, d'Armando Petrucci ${ }^{2}$. Il s'agit de proposer un regard différent de celui généralement admis par la tradition historiographique selon laquelle l'écrit est vu comme un élément constitutif d'un objet donné et non comme une réalité culturelle globale.

En feuilletant les manuels de paléographie, des plus anciens aux derniers publiés, on constate que la discipline s'intéresse presque exclusivement aux écritures des livres et des chartes: un exemple représentatif est dans l'introduction du manuel de Maurice Prou, un des pères de la paléographie française :

"La paléographie est la science des anciennes écritures. Elle a pour objet le déchiffrement des écritures de l'antiquité et du moyen âge. Son domaine s'étend à toutes sortes de documents écrits : inscriptions, monnaies, sceaux, actes et livres. [...] Nous laisserons à côté les inscriptions, les légendes des monnaies et celles des sceaux : leur étude regarde plus spécialement l'épigraphie, la numismatique, la sigillographie. On s'en tiendra donc ici à l'étude et au déchiffrement des monuments de l'écriture tracés sur papyrus, sur parchemin ou sur papier"'3.

Selon la tradition résumée par l'auteur, les sciences auxiliaires de l'histoire se divisent sur la base de la nature de la pièce archéologique; mais seulement la codicologie et la diplomatique ont le privilège de recevoir les faveurs de la paléographie, parce que le livre et la charte sont les porteurs d'écriture par excellence même si Prou déclare clairement son implication dans les autres disciplines nommées. D'ailleurs encore aujourd'hui, en parlant de paléographie on fait référence à

1980. "Epigrafia e paleografia. Inchiesta sui rapporti tra due discipline", dans Scrittura e civiltà, 5, 1981.

2 "L'oggetto di studio della paleografia è ciascuna testimonianza scritta a mano, dalle origini della scrittura, fino alla stampa": A. PETRUCCI, Lezioni di storia della scrittura latina, 1985, p. 6.

${ }^{3}$ PROU Maurice, Manuel de paléographie latine et française du VI au XVII siècle, suivi d'un dictionnaire des abréviations avec 23 fac-similés en phototypie, Paris, Alphonse Picard éditeur, 1890, p. XI. 
la science qui s'occupe de l'étude des écritures anciennes en général, mais qui, en réalité, s'attache presque exclusivement au seul domaine du livre manuscrit. Les lettres présentes sur les supports différents du papyrus, du parchemin ou du papier (principalement la pierre, plutôt que le métal ou le bois...), n’ont pas encore reçu un traitement qui permette d'en établir les singularités éventuelles et leur évolution historique. Pour en proposer une analyse, souvent trop brève et superficielle, on fait toujours appel aux notions et à la terminologie de cette paléographie, qu'on pourrait définir livresque, en oubliant que "Paleografia y Epigrafia son ciencias que tienen un objeto común de estudio: la escritura. Y como acontece a la Paleografia, la Epigrafia no reduce su ambito al desciframiento, datación y localización, sino que es parte de la historia de la cultura" ${ }^{\text {. }}$.

C'est sur cette proposition que la recherche engagée au sein du Centre d'études supérieures de civilisation médiévale (CNRS - Université de Poitiers) et présentée rapidement dans cet article pose ses fondements. Son objectif est de réévaluer l'écriture au sein de la société médiévale où l'écrit acquiert non seulement une valence fonctionnelle importante, mais aussi un sens symbolique très fort. $\mathrm{Au}$ niveau pratique, on décrira ici la méthodologie choisie pour avancer dans ce type d'analyse (méthodologie en cours de texte, d'où son caractère encore largement empirique), les outils existants ou à créer nécessaires pour le travail, ainsi que les difficultés face auxquelles on se trouve en empruntant ce nouveau sentier.

L'objectif ultime de cette étude serait de proposer un parcours systématique sur l'évolution des formes des lettres lapidaires identique à ce que l'on utilise déjà pour l'analyse des graphies manuscrites, parcours permettant à la paléographie de devenir un critère de datation épigraphique plus fiable scientifiquement et dépassant les constats de similitude et de variation ${ }^{5}$. Pour cela, la démarche entend conduire une étude comparée entre l'écriture d'apparat des codices et celle des inscriptions (en cherchant, si possible, d'inclure aussi d'autres objets, sceaux et mon-

${ }^{4}$ L. NÜÑEZ CONTRERAS, Manual de paleografía: fundamentos e historia de la escritura latina hasta el siglo VIII, Madrid, Catedra, 1994, p. 24.

${ }^{5}$ Comme souligné aussi par I. DI STEFANO MANZELLA, Mestiere di epigrafista. Guida alla schedatura del materiale epigrafico lapideo, Roma, Edizioni Quasar, 1987, p. 135: "L'esame della tecnica scrittoria e della grafia mira da una parte a fornire risultati pratici immediati [...] dall'altra a segnalare l'esistenza di fenomeni che interessino la Paleografia, ossia la disciplina che si occupa della storia delle forme grafiche". Même si plutôt daté, encore aujourd'hui il s'agit du manuel d'épigraphie de par excellence pour le domaine italien. 
naies spécifiquement), afin de présenter, expliquer, tester une méthode claire, simple et efficace, applicable dans l'avenir à d'autres corpus ${ }^{6}$.

\section{LA MÉTHODE.}

L'étape préliminaire à accomplir dans cette démarche consiste à choisir le matériel sur lequel travailler ; celui-ci doit constituer a priori un corpus unitaire. La constitution de cet ensemble documentaire valide, avec des caractéristiques intrinsèques susceptibles de poser des fondations solides pour les résultats de la comparaison, est à la base de l'étude entière. Les critères reposent sur la quantité et l'unité du corpus ; il est en effet nécessaire de choisir un matériel qui soit non seulement le plus homogène du point de vue de la provenance et de la chronologie, mais il doit être également suffisamment riche que la comparaison soit statistiquement recevable.

La collection des témoignages graphiques de la ville de Toulouse, déjà examinée pour la partie épigraphique au sein de notre travail de Master à l'Université de Venise, et donc partiellement connue, constitue un laboratoire idéal pour le type d'investigation en cours : l'homogénéité donnée par l'origine limitée à la ville en général et à trois sites en particulier, le nombre suffisamment élevé de pierres et de manuscrits et le milieu culturel dans lequel la société évolue pendant les siècles choisis $\left(\mathrm{XI}^{\mathrm{e}} \mathrm{XIV}^{\mathrm{e}}\right)$. Pour la partie manuscrite, la constitution du corpus n'est pas encore définitive, mais les critères requis sont les mêmes que ceux retenus pour l'ensemble épigraphique (cohérence et abondance), auxquels il faut ajouter la présence d'écriture d'apparat. La comparaison est en effet réalisable pour des graphies de la même "famille" et les écritures utilisées pour les titres et les rubriques sont les plus proches à celles des inscriptions gravées ${ }^{7}$.

Une recherche épigraphique satisfaisante commence par la connaissance de l'objet et l'établissement du corpus augmenté du plus grand nombre d'informations relatives aux documents. Le stade suivant consiste en la compilation des fiches descriptives, une pour chaque pièce analysée. La fiche se compose

${ }^{6}$ Une référence explicite à cette "méthode comparative également utilisée par les paléographes" on la trouve dans C. TREFFORT, Paroles inscrites. À la découverte des sources épigraphiques latines du Moyen Âge, Rosny-sous-Bois, Bréal éditions, 2008, p. 32.

${ }^{7}$ V. GARCIA LOBO, "La escritura publicitaria en la Peninsula Ibérica. Siglos X-XIII", dans Inschrift und Material, Inschrift und Buchshrift, éd. Scientifique par C. KOCH, C. STEININGER, München, 1999; P. STIRNEMANN, M. SMITH H., "Forme et fonction des écritures d'apparat dan les manuscrits latins (VIII $-\mathrm{XV}^{\mathrm{e}}$ siècle) “, dans Bibliothèque de l'école des chartes, $\mathrm{t} .165$, 2007, p. 67-100. 
de différentes sections qui témoignent d'une recherche en profondeur sur chaque inscription : une première partie recueille les données relatives à la localisation (lieu d'origine, lieu de conservation, notes sur la provenance et la topographie), une autre les aspects matériels (support et description de l'organisation de l'écriture) ; la dernière section est dédiée au texte (transcription, édition, traduction). La création d'une base de données est toujours envisagée pour gérer et manipuler rapidement cette grande quantité d'information sur de nombreuses pièces. Dans la mesure où elle contient tous les détails descriptifs de l'objet, la recherche permet plusieurs actions : trier par chronologie, provenance, type d'écriture ou de support, typologie textuelle ; chercher un certain ouvrage à travers le nom d'un personnage qui y est mentionné, plutôt que par le nom du scribe ; utiliser des mots clés pour arriver plus vite à un document individuel ou à un groupe de documents. Toutes ces méthodes permettent sans aucun doute de gagner du temps grâce à une interrogation agile d'un vaste corpus, mais surtout de générer de nouvelles questions en questionnant un ensemble riche et varié. Soulignons enfin que chaque fiche doit être accompagnée d'une ou plusieurs photographies du document, réalisées sur le terrain, qui permettront la véritable analyse comparative.

Pour les manuscrits, l'opération est à première vue simple dans la mesure où l'on peut "manipuler" le codex, le déplacer, régler la lumière, etc. Les inscriptions, par contre, présentent toute une série de difficultés majeures : leur emplacement peut limiter l'accès ; sauf exception, elles ne peuvent pas être déplacées, la lumière n'étant donc pas facile à traiter; à cela s'ajoutent les gênes dues à la pierre, à la profondeur du trait ou encore aux conditions de conservation. On détermine deux étapes successives et fondamentales dans la création de la partie figurée du dépouillement : la photographie et le dessin. L'épigraphiste qui décide de s'occuper de la campagne photographique des plaques, lui-même ou à l'aide d'un professionnel, devra tout d'abord avoir lu et compris l'inscription. La compréhension de l'objet et de sa partie textuelle est en fait le fondement pour arriver à avoir des reproductions utilisables : les signes non alphabétiques, les détails importants, les lettres particulières ne peuvent pas être distinguées avant une bonne assimilation. Dans la plupart des cas ces parties demanderont des attentions spéciales (au niveau de lumière, prise de vue, position...) pour être bien reproduites ; des choix pas toujours esthétiquement jolies satisfaisants, mais scientifiquement déterminants. Une bonne photographie est indispensable pour travailler facilement au stade suivant, celui du dessin, capable de reproduire une image fidèle de l'objet original. Le dessin " à main levée " est à proscrire et on lui préfèrera celui qu'en 
épigraphie on appelle relevé de l'inscription, de façon manuelle ou à l'aide de logiciels dédiés.

Pour le relevé manuel, après avoir nettoyé la pierre avec un pinceau, il faut couvrir l'inscription avec du papier transparent (le plus adapté étant le papier qu'utilisent les fleuristes), le fixer par du ruban adhésif en papier et procéder au décalque de l'écriture avec des crayons fins. Cette méthode est très utile parce qu'à l'aide d'une lumière rasante elle permet d'isoler le trait constitutif du signe alphabétique de l'enchevêtrement des autres traits de la pierre abîmée ; dans la mesure où elle s'effectue par contact, cette technique n'est pas toujours possible. La démarche alternative consiste alors à calquer le texte à partir d'une photo ; dans ce cas, on a la possibilité d'utiliser des programmes informatiques qui permettent d'élaborer les photos et d'obtenir des résultats très facilement exploitables.

Le procédé qu'on vient de décrire exige une série d'observations : le relevé fait à la main à partir d'une photo est un processus plus facile pour l'épigraphiste (surtout si les inscriptions sont conservées dans des endroits difficiles d'accès) et plus sûr pour les pierres (quand elles sont abimées); que le relevé soit fait d'une façon manuelle ou informatique, il doit toujours être accompagné par la photographie, car rien ne la surpasse pour la compréhension de l'objet. La technique de calque de l'écriture ici décrit pour les textes gravés sera le même utilisée pour la reproduction de l'écriture des manuscrits ${ }^{8}$.

Si en général le dessin, dans chaque domaine, aide à fixer la forme de l'objet représenté, dans ce cas, il assume une importance fondamentale, puisqu'il est au cœur du processus même d'analyse des inscriptions : c'est à partir du relevé que le tableau paléographique est ensuite construit. Instrument par excellence de cette démarche, il s'agit simplement d'un tableau qui contient les alphabets employés dans le texte examiné : on recueille la variété graphique pour chaque lettre de chaque objet contenant l'écriture et on les dispose dans cette grille. Les critères de classement internes au tableau sont chronologiques et en fonction de la provenance de l'objet : on cherchera à grouper les objets provenant de la même aire géographique et, à l'intérieur de l'ensemble crée, de les mettre en ordre chronologique (un exemple à la figure 1). Dans toutes les études paléographiques, des plus anciennes aux modernes, la présence des tableaux paléographiques confirme l'importance d'une analyse comme celle-ci de son impact visuel, capable d'aller

\footnotetext{
${ }^{8}$ Dans le cas de la reproduction de l'écriture d'apparat des manuscrits, le travail à partir du cliché photographique est encore plus conseillé, pour garder l'intégrité du parchemin.
} 
bien au-delà de la meilleure des descriptions écrites d'une lettre. Cet outil est le plus efficace pour établir une étude scientifique, au-delà des impressions visuelles, des variations de l'écriture dans la mesure où il permet de synthétiser tous les changements morphologiques des lettres en même temps, de procéder à la comparaison et d'essayer d'avancer enfin une proposition de leur histoire évolutive (figure $2^{9}$ ).

\section{DIFFICULTÉS.}

Cette démarche, encore toute à créer, présente des avantages, mais fait également état de difficultés réelles, dont la première consiste en le manque d'outils adéquats pour mener à bien cette approche nouvelle.

Face à la description de la lettre gravée, le premier obstacle saute aux yeux : l'absence d'un vocabulaire paléographique spécifique pour l'écriture gravée. La terminologie utilisée jusqu'à ce moment est celle reprise du domaine du livre manuscrit, qui ne peut pas toujours couvrir les exigences descriptives de l'épigraphie en même temps ainsi qu'elle crée des malentendus ou des rapprochements abusifs, sans parler des formes des lettres ambigües, voir spéciales, qui exigent parfois un effort de fantaisie de la part de l'épigraphiste. Pour combler cette lacune, une des parties les plus importantes de notre recherche consiste à la création d'un éventail de mots techniques pour décrire, de la façon la plus simple et la plus efficace, les formes spécifiques des écritures non livresques. À partir des manuels paléographiques traditionnels, on cherchera a modeler les définitions déjà existantes. La constitution d'un lexique paléographique global se présente à la fois comme une opération simple, puisqu'on peut faire recours à la terminologie traditionnelle et parce qu'on doit décrire un nombre mineur de typologies graphiques par rapport au domaine manuscrit, mais il s'agit aussi d'un travail extrêmement délicat qui doit être capable de décrire une lettre simplement, mais en soulignant les differentes techniques qui sont à la base de la réalisation de l'écrit.

Les paléographes, par exemple, parlent de "pleins et déliés" pour désigner le jeu de plume qui crée des traits plus épais ou plus fins, selon le mouvement de la pointe sur le support ; ce phénomène plus ou moins marqué est naturellement présent sur le folio du manuscrit médiéval, comme résultante de l'outil d'écriture. Dans les inscriptions d'un niveau qualitatif élevé, on trouve la même manifesta-

\footnotetext{
${ }^{9}$ Elle représente, l'une après l'autre, les trois étapes de l'étude de l'inscription : la photographie (1), le relevé (2) et le tableau paléographique (3).
} 
tion, mais dérivant celle fois d'une volonté spécifique du lapicide : ce n'est pas la forme de la pointe qui passe sur la pierre qui détermine le jeu de lumière, mais il s'agit d'un choix précis de l'artiste d'épaissir certains traits, en en laissant d'autres plus subtiles (un exemple à la figure). Cet artifice, loin d'être un effet naturel et absolument absent dans les inscriptions plus modestes, doit être toujours remarqué dans une description de l'écriture épigraphique, en tant que caractéristique représentative d'une qualité productive capable d'amener à une histoire sociale de l'inscription.

La liste des questions sur la terminologie peut devenir rapidement très longue: comment distinguer une conjonction d'un enclavement ou encore d'un entrelacement? Peut-on utiliser le mot d' " empattement " pour décrire les éléments décoratifs à la fin d'une haste, ou ce mot risque de créer de malentendu avec sa signification en typographie ? Selon quels critères définir le niveau d'habileté du lapicide? Il s'agit ici, à mon avis, d'une série de questions légitimes, dont les réponses demandent un travail de réflexion, exigent d'être testées puis validées avant de les intégrer au travail quotidien.

La carence de bibliographie est la deuxième lacune plutôt importante. Pour ce qui concerne l'investigation autour de la paléographie lapidaire, on a déjà fait allusion à la littérature spécifique datée, insuffisante ou limitée à des cas ponctuels, trop spécifiques pour servir de base à une réflexion générale.

On doit la première tentative de tracer une histoire évolutive de l'écriture épigraphique à Paul Deschamps ${ }^{10}$ : le médiéviste a cherché à reparcourir les transformations de l'écriture à l'intérieur d'un ensemble trop vaste, qui manque pourtant d'homogénéité au niveau géographique, négligeant ainsi l'un des principes fondamentaux de ce type d'analyse ${ }^{11}$. Comme dit Robert Favreau à propos de cette contribution, “ il convient donc de ne pas utiliser l'étude de P. Deschamps et

${ }^{10}$ P. DESCHAMPS, "Étude sur la paléographie des inscriptions lapidaires de la fin de l'époque mérovingienne aux dernières années du XIIe siècle”, extrait du Bulletin monumental, Paris, 1929

${ }^{11}$ L'objectif de l'auteur était celui de proposer une approche différente par rapport aux études précédentes; "[...] le champ de leurs études était trop étroit et le nombre des inscriptions examinées pour chaque siècle trop restreint, pour leur permettre de concevoir dans ses grandes lignes l'évolution des caractères paléographiques des inscriptions lapidaires pendant la période médiévale" il dit explicitement à page 6, en référence aux travaux comme Manuel d'épigraphie suivi $d u$ recueil des inscriptions du Limousin par M. Textier; épigraphie du Haut-Poitou, par M. DE LONGUEMAR, etcetera. 
ses tableaux de lettres comme une référence absolue, ce qui est une tentation car il n'y a pas d'autre synthèse générale “ 12 .

Dans les dernières années dans la collection de l'École des chartes a paru une autre synthèse, rédigée selon des critères modernes, et qui pour l'instant e reste le seul et irremplaçable point de référence ${ }^{13}$ : même si les auteurs invitent le lecteur à voir surtout les aspects culturels et sociaux de l'inscription, ils le font à travers une analyse de l'écriture claire et efficace.

Sans jamais oublier ce travail essentiel, une attention spéciale doit être réservée aux études de cas, car ils pourraient se révéler des bons points de départ pour une histoire de l'écriture plus vaste ${ }^{14}$. De ce point de vue les exemples sont nombreux et dispersés sur l'ensemble du territoire de la France actuelle, mais je voudrais proposer spécifiquement de traiter ici l'étude de Chantal Fraïsse ${ }^{15}$ : l'historienne de l'art, en analysant le programme iconographique du cloitre de Saint-Pierre de Moissac, offre une comparaison entre la structure architecturale du cloitre et la production écrite. Pour la partie qui me concerne plus strictement, elle rapproche la structure de l'inscription du pilier à celle des incipit et explicit des manuscrits et l'ensemble du programme iconographique des chapiteaux aux images des enluminures des codices. Sans rentrer trop dans le détail du contenu, le mérite de cette contribution est double : d'une part celui de lier le domaine du livre à celui de l'épigraphie (et de la sculpture), en argumentant de manière exemplaire l'influence réciproque des deux sphères ; d'autre côté, du point de vue descriptif, le lexique utilisé pour parler de l'écriture est à la fois scientifique et extrêmement simple.

À partir de ce point de vue, on peut souligner la double valence de la contribution de la paléographie dans ce type de recherche : pour la perspective métho-

12 R. FAVREAU, Épigraphie médiévale, Turnhout, Brepols, 1997. Même s'il s'agit d'un livre apparu il y a quinze ans désormais, l'affirmation de l'auteur reste toujours actuelle.

${ }^{13}$ V. DEBIAIS, R. FAVREAU, C. TREFFORT, 'L'évolution de l'écriture épigraphique en France au Moyen Âge et ses enjeux historiques", dans Bibliothèque de l'école des chartes, t. 165, 2007, p. 101-137.

${ }^{14}$ Je suggère, parce que particulièrement éclairants sur les influences réciproques entre domaine manuscrit et épigraphique, les deux articles suivants: I. WEINRY, The inscribed image: negotiating sculpture on the coast of the Adriatic Sea, Word \& Image: A Journal of Verbal/Visual Enquiry, 2011, disponible sur internet: http://dx.doi.org/10.1080/02666286. 2011.541133; J. O'DRISCOLL, Visual vortex: an epigraphic image from an Ottonian gospel book, Word \& Image: A Journal of Verbal/Visual Enquiry, Volume 27, Issue 3, 2011, Special Issue: The Iconicity of Script: Writing as Image in the Middle Ages, p. 309-321.

${ }^{15}$ C. FRAÏSSE, "Le cloitre de Moissac a-t-il un programme?", dans Cahiers de civilisation médiévale, $X^{e}-X X I I^{e}, 50^{\mathrm{e}}$ Année - Juillet-Septembre, p. 245-270. 
dologique, en se posant comme base de la création d'une terminologie épigraphique spécifique; d'un autre côté en référence à l'histoire culturelle, pour la comparaison des formes des écritures d'apparat manuscrites que l'on peut retrouver dans les inscriptions gravées. Dans un même ordre d'idée, le rapprochement possible entre les titres du Rouleaux des morts de Bosone ${ }^{16}$ concernant son passage à Toulouse et certaines inscriptions conservées au Musée des Augustins est exemplaire : en regardant certains détails des figures 4 et $5^{17}$, (comme par exemples la forme cursive de la lettre E de Aldemare dans l'inscription et du mot Tolose dans le titulus du rouleau) ou en général les jeux de lettres présentes aussi bien sur la pierre que sur le parchemin, résulte plutôt facile s'apercevoir qu'il s'agit du même style d'écriture, quoique dans des supports différents ${ }^{18}$.

\section{EN PERSPECTIVE.}

Dans son Épigraphie médiévale, Robert Favreau, au chapitre consacré à la paléographie des inscriptions, attire l'attention sur une série d'éléments intéressants. Tout d'abord il fait référence à la notion d' " ensemble homogène " sur lequel effectuer l'analyse paléographique, car la géographie et la période de provenance d'une inscription déterminent profondément ses caractères internes : c'est pour ça qu'il envisage de procéder par "études systématiques [...] de villes ou de régions “. Il souligne ensuite qu'il faut avoir une attention spéciale pour le support et pour l'environnement culturel dans lequel l'artiste lapicide opérait, éléments qui déterminent la technique et le résultat des formes graphiques. Enfin, il fait une petite esquisse du problème du vocabulaire descriptif et il précise que " on évitera des termes trop compliqués et on expliquera les termes techniques retenus ".

À travers les mots du fondateur de l'épigraphie médiévale en France, on peut simplement parcourir l'idée générale de cette recherche. Ce que je voudrais ici proposer, c'est en fait l'explication détaillée d'une méthode, dont les démarches sont déjà pour la plupart connues et utilisées systématiquement par les épigraphistes italiens. Il s'agit donc de les adapter aux exigences locales, les affi-

${ }^{16}$ M. P. NICCOLI, "Il rotolo funerario di Bosone, abate di San Giusto di Susa. Edizione critica", dans La Novalesa. Ricerche, fonti documentarie, restauri, vol. II, Archivio di Stato di Torino, 1981, p. 77.

${ }^{17}$ M. P. NICCOLI, “Il rotolo funerario di Bosone...", Tav. II.

18 Pour une analyse complète et efficace, il faudrait procéder avec les tableaux paléographiques, à confirmer quant dit jusqu'ici à propos de leurs importance, si non nécessité. 
ner si nécessaire et les tester au sein d'une équipe de chercheurs de formation différente, permettant d'envisager la globalité du phénomène graphique ${ }^{19}$.

Cette méthodologie sera mise à l'épreuve du corpus (épigraphique et manuscrit) presque idéal de la ville de Toulouse. Le caractère exceptionnel de ses collections est dû à leur sérialité, cette caractéristique de cohérence envisagée par Robert Favreau.

On cherchera à pourvoir au manque d'outils de travail, à partir du vocabulaire, afin de pouvoir parler tous la même " langue épigraphique ", qui permettra de rédiger des descriptions paléographiques harmonieuses au niveau national, de se faire comprendre facilement par des spécialistes comme par des amateurs, par des Français comme par des étrangers. Nous souhaitons également promouvoir parmi les épigraphistes l'usage du relevé des inscriptions et du tableau paléographique : le premier s'est souvent montré non seulement le moyen le plus efficace pour obtenir la restitution des textes, même les plus abimés, et donc difficiles à lire, mais aussi l'outil essentiel pour la constitution des tableaux paléographiques, méthode permettant la comparaison entre plusieurs inscriptions.

Le travail est ambitieux, sans aucun doute, mais il s'inscrit dans un mouvement général de coopération académique entre les épigraphistes européens ${ }^{20}$, entre chercheurs des différentes disciplines de l'érudition et, de façon plus large, entre spécialistes des sciences humaines et des sciences pour l'ingénieur ${ }^{21}$. Il est donc tout à fait réaliste d'envisager d'ici à la fin de ma thèse des résultats intéressants, mais surtout utiles pour le processus évolutif dans le sens de cette interdisciplinarité, jusqu'ici autant acclamée que gardée soigneusement loin, la seule capable d'apporter une lumière nouvelle sur la notion d'écriture médiévale.

\footnotetext{
${ }^{19}$ J'ai la chance de travailler au Centre d'études supérieures de civilisation médiévale (CNRS - Université de Poitiers) et de pouvoir profiter de la collaboration et des conseils d'une équipe d'épigraphistes et d'aspirants épigraphistes, dont la richesse réside dans les différents points de vue par lesquels on regarde l'inscription.

${ }^{20}$ Grâce à la série d'initiatives européennes encouragées surtout par l'institution du Comité international d'épigraphie médiévale et moderne, dans lesquelles l'université de Poitiers est participe spécialement.

${ }^{21}$ Dans les dernières années sont nées plusieurs projets qui lient informaticiens et humanistes autour de l'élaboration et la reconnaissance informatique de l'écriture médiévale: voir par exemple, le programme NUID-CARACO, que le C.E.S.C.M. partage avec le laboratoire SIC sur la caractérisation automatique des formes épigraphiques, et celui envisagé avec l'IRHT dans le cadre d'un appel d'offres de l'ANR qui fera suite au programme Graphem.
} 
EVA CARAMELLO

PALEOGRAPHIE COMPAREE DES ECRITURES EPIGRAPHIQUES ET MANUSCRITES :

UNE METHODOLOGIE EN EVOLUTION

IMAGES

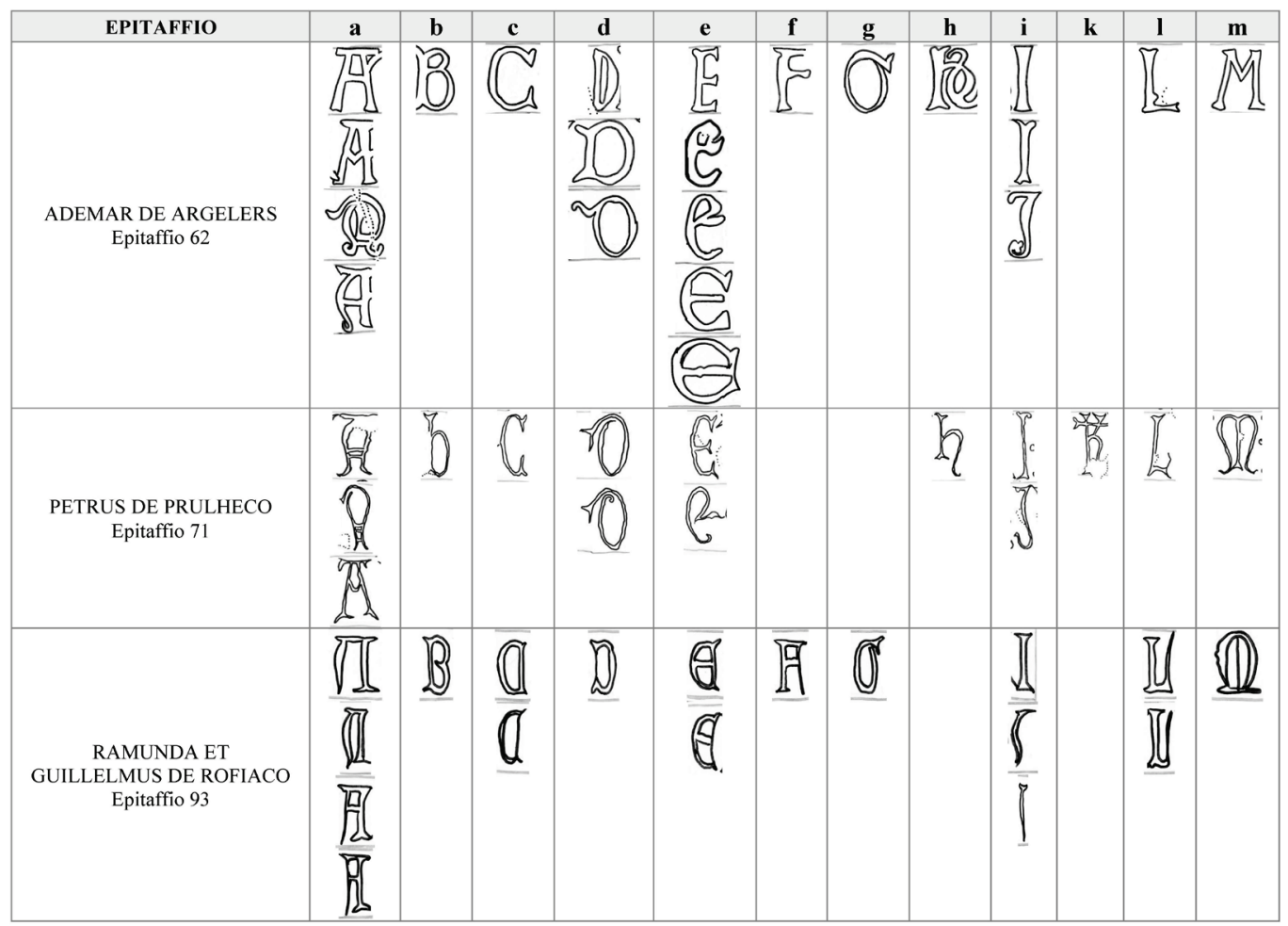

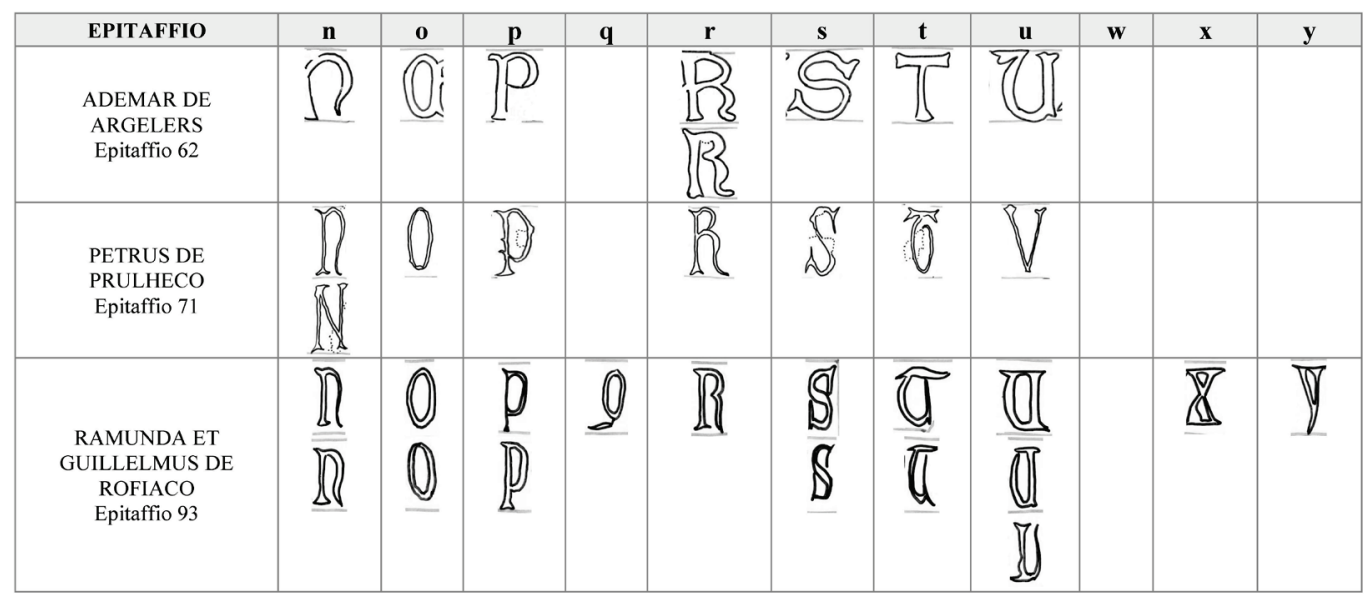

Figure 1. Exemple de tableau paléographique. 


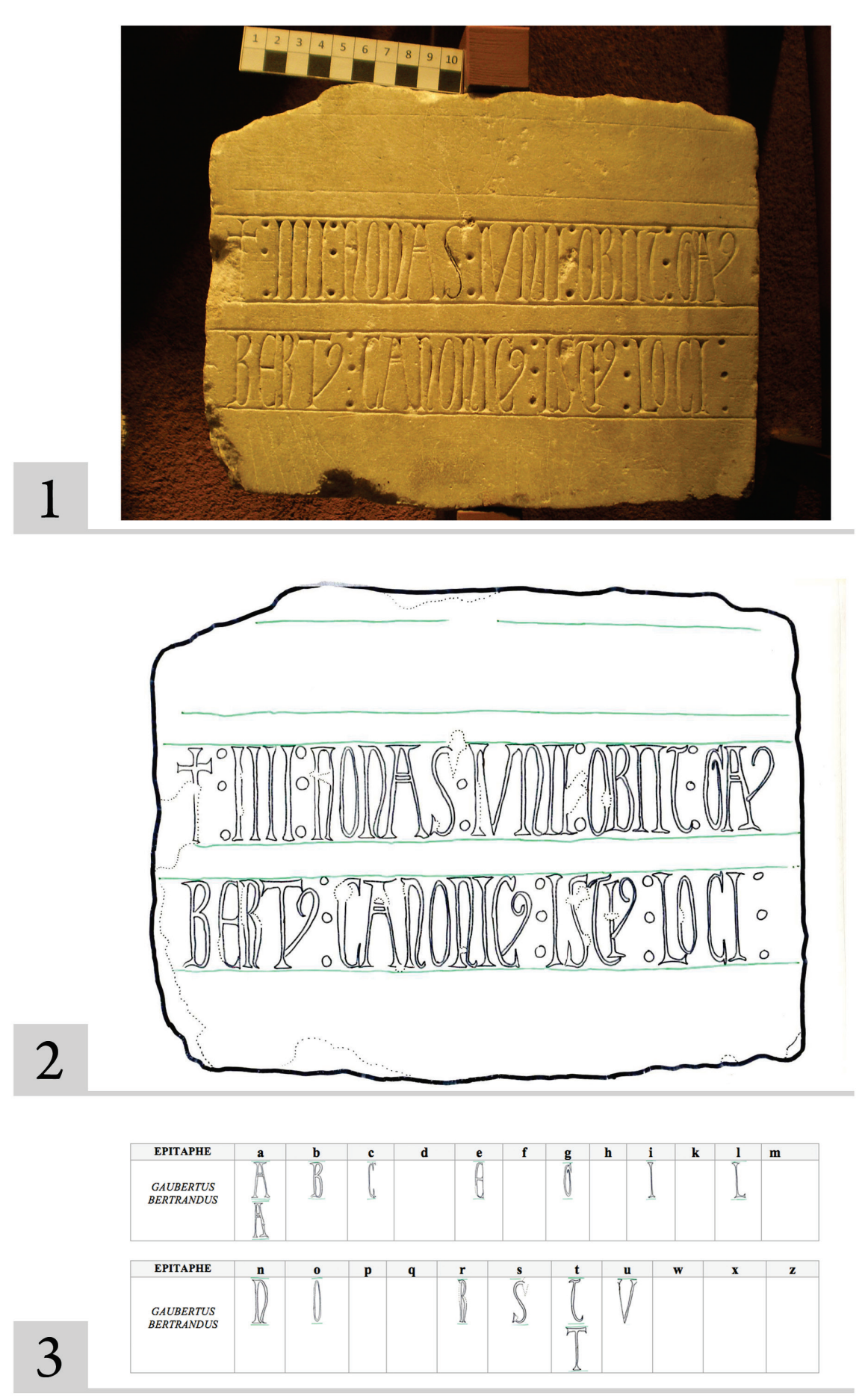

Figure 2. Les de l'étude de l'inscription: la photographie (1), le relevé (2) et le tableau paléographique (3). Epitaphe de Gaubertus, Corpus des Inscriptions de la France médiévale, vol.7, Toulouse, $\mathrm{n}^{\circ}$ 104, p.144. (Cliché Eva Caramello) 
PALEOGRAPHIE COMPAREE DES ECRITURES EPIGRAPHIQUES ET MANUSCRITES : UNE METHODOLOGIE EN EVOLUTION

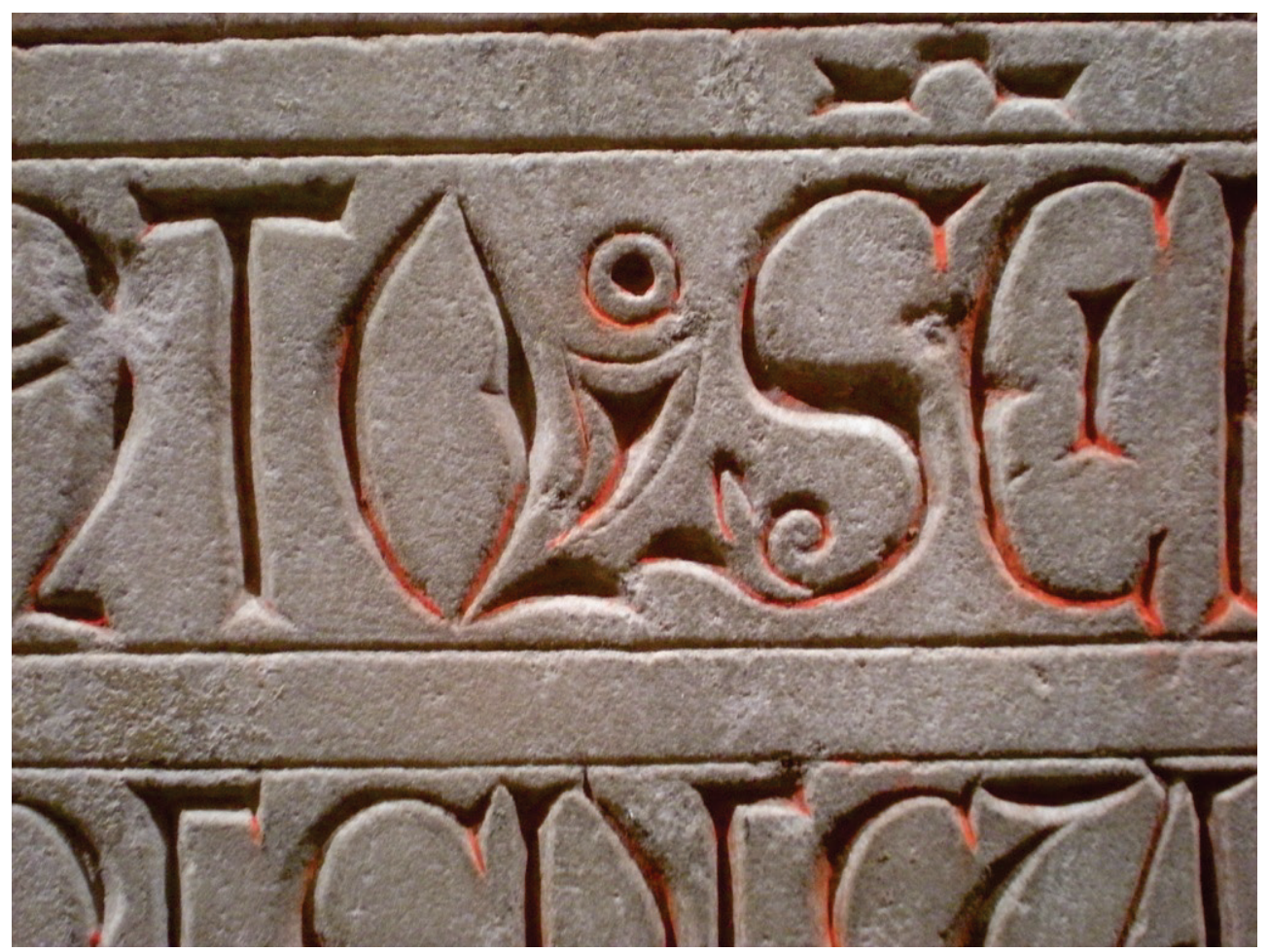

Figure 3. Détail du jeu de lumière dans l'épitaphe de Petrus Bernardi, Corpus des Inscriptions de la France médiévale, vol.7, Toulouse, n50, p.90. (Cliché Eva Caramello)

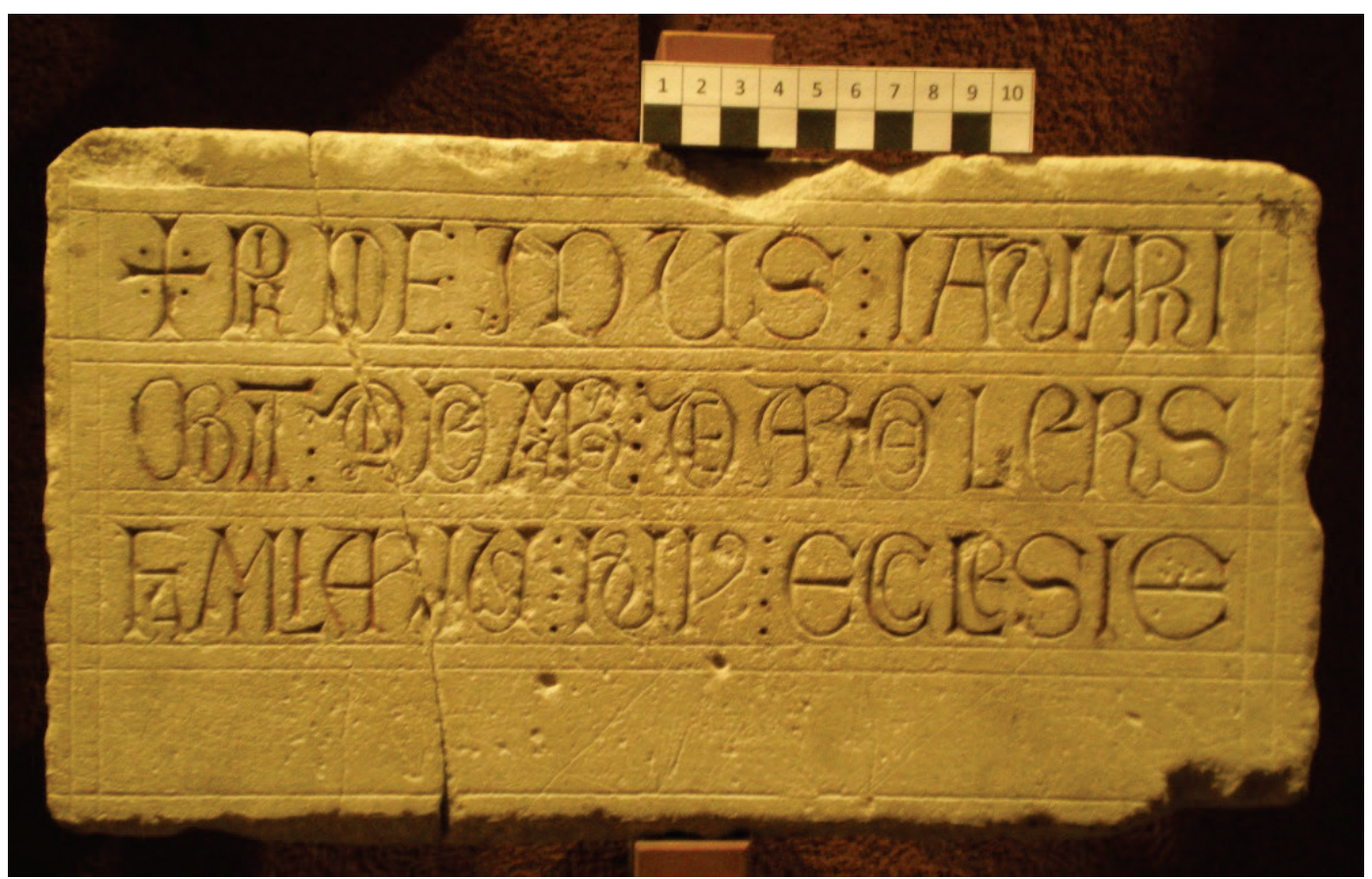

Figure 4. Epitaphe de Aldemarus de Argelers, Corpus des Inscriptions de la France médiévale, vol.7, Toulouse, n62, p.102. (Cliché Eva Caramello) 


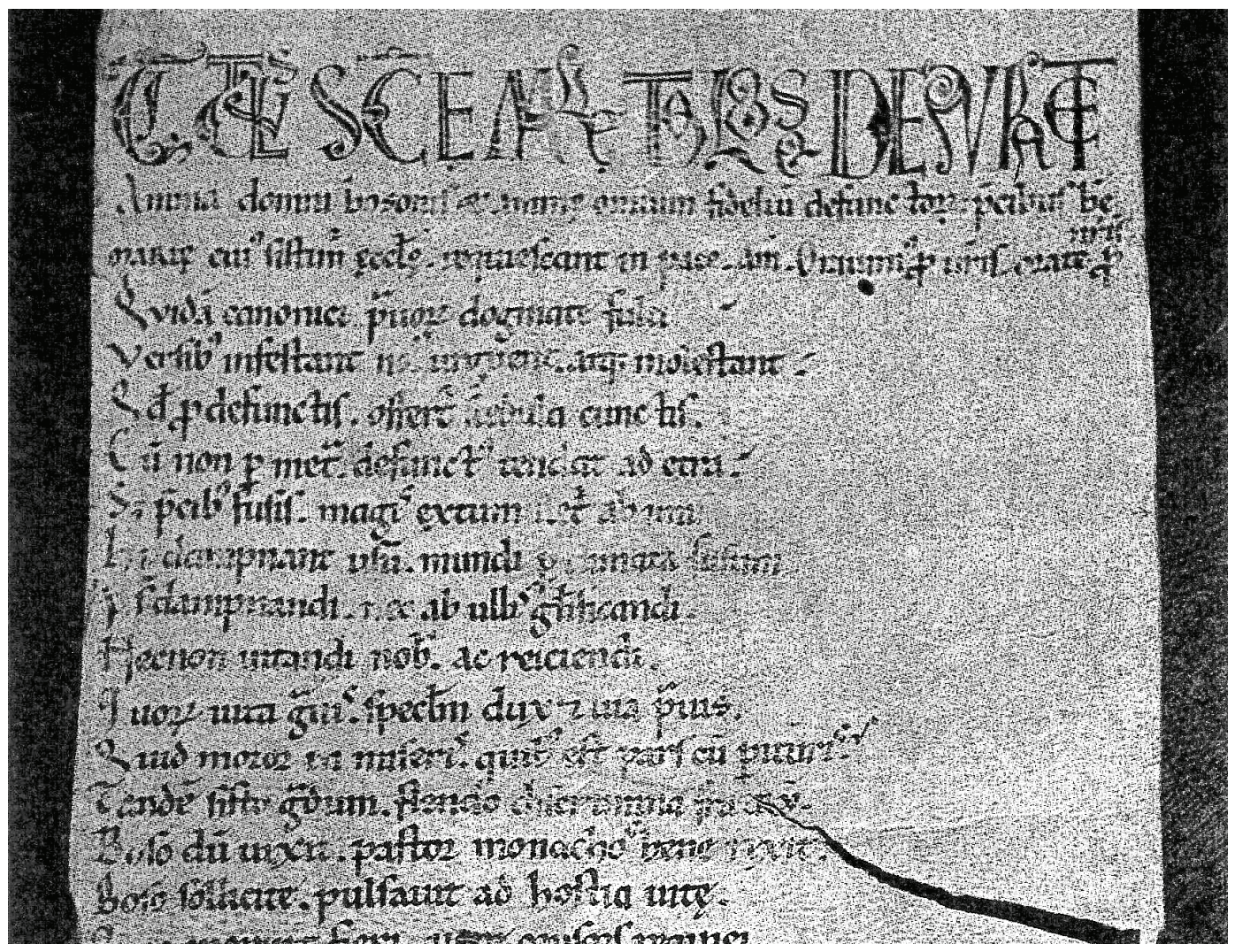

Figure 5. Rouleau funéraire de Bosone. 